\title{
An evaluation method of urban epidemic emergency response capability based on fuzzy logic
}

\author{
$X u$ Cheng $^{1}$, Yanyan Huang ${ }^{1, *}$, and Peiting $\mathrm{Wu}^{1}$ \\ ${ }^{1}$ School of Automation, Nanjing University of Science \& Technology, Nanjing, China
}

\begin{abstract}
In 2020, the outbreak of COVID-19 seriously threatened many people's life and it had brought unexpected impacts upon the global economy. In order to understand the emergency response ability of cities with similar backgrounds, an evaluation method based on fuzzy comprehensive evaluation was proposed. First, we established the evaluation index system of urban epidemic response capability. Meantime, a combination way to calculate the index weights of the evaluation system was developed based on analytic hierarchy process. Finally, we have an empirical analysis on the evaluation activity of epidemic emergency management in area $\mathrm{H}, \mathrm{J}$ province. The evaluation results can be used as the basis for selecting advanced cities. At the same time, it is of great importance for area $\mathrm{H}$ to improve urban resource allocation and emergency response capability.
\end{abstract}

\section{Introduction}

Since the 21st century, major outbreaks including SARS, influenza A-H1N1, avian influenza (H7N9) and COVID-19 all had characteristics such as violent, destructive and unpredictable, which brought challenges and major tests on our epidemic prevention. Since the outbreak of coronavirus, China has suffered from huge economic losses even though the government has actively applied several effective measures.

After this epidemic, every government need to consider how to improve the city's emergency response capability. Region $\mathrm{H}$ of province $\mathrm{J}$ has specially carried out an evaluation among advanced cities for their epidemic emergency management. Different cities have their own advantages and disadvantages on emergency management thus makes it difficult to compare. Therefore, a reasonable and fair evaluation method is urgently needed. How to evaluate the emergency response capability will be the main focus of this study.

Consider that there are many fuzzy indices on the preparedness capability of the urban epidemic, we have to resort to a kind of method which can deal with such fuzzy description. In recent years, many scholars have carried out research on fuzzy comprehensive evaluation ${ }^{[1-3]}$. In the field of epidemic prevention and control, there are some references about such research results ${ }^{[4-5]}$. There are some advantages to adopt such fuzzy comprehensive evaluation. Therefore, we decide to evaluate the urban epidemic emergency response capability based on fuzzy comprehensive evaluation.

* Corresponding author: imhyy@sina.com 


\section{Assessment model of epidemic emergency response capability}

\subsection{Epidemic emergency response capability}

In this paper, the definition of urban epidemic emergency response capability is derived from the meaning of combat capability in military field. According to the analysis of relevant references, this paper gives the definition of urban emergency response capability as: in the process of an outburst epidemic situation, the capability measurement of the cities to fight with the epidemic situation.

According to the above-mentioned definition of emergency response capability, we find that what we need to do is to find a way to quantify emergency response capabilities. In order to evaluate the emergency response capability of each city, the following key issues need to be solved. Firstly, the scenario of sudden epidemic situation; secondly, the construction and general description of the emergency capability index system; finally, the evaluation method of emergency capability value. Following, the above problems will be solved one by one.

\subsection{Evaluation method modeling}

\subsection{1 scenario of epidemic evaluation}

It is usually assumed that on a certain day in a certain year, a number of cities in $\mathrm{H}$ area of $\mathrm{J}$ province have a major infectious disease, which is highly infectious and could kill some people. Therefore, the local government is required to carry out emergency control to prevent the spread of the epidemic. The regional government responded to the epidemic by mobilizing medical personnel, collecting medical materials and restricting the flow of personnel.

There are differences in the aspects of government governance, urban construction and public awareness between different cities. In the assessment of emergency response capability, it is necessary to aggregate the basic capabilities if we want to evaluate the advanced level of each city reasonably.

\subsubsection{Index system for assessing the epidemic response capability}

To establish the evaluation model of urban epidemic response capability, it is necessary to establish the corresponding evaluation index system first. In general, an assessment index system as shown in Figure 1 can be established for the assessment of epidemic response capability. For region $a, E^{a}$ represents the assessment value of epidemic response capability of region $a$. Corresponding to the evaluation index system in Figure 1, mathematical formal symbols are used to describe the relationship between the evaluation indices of emergency capability, and the results are shown in Figure 2. $U_{i}^{a}$ represents the capability value of the i-th composite index of emergency capability, which is a composite function of the sub-evaluation index parameter $x_{i j}$. In general, emergency capability assessment indices can be decomposed. Fuzzy evaluation is carried out from the sub-index, combined with the corresponding weight value, and fuzzy synthesis is carried out to the upper layer gradually, until the final composite evaluation value is obtained. 


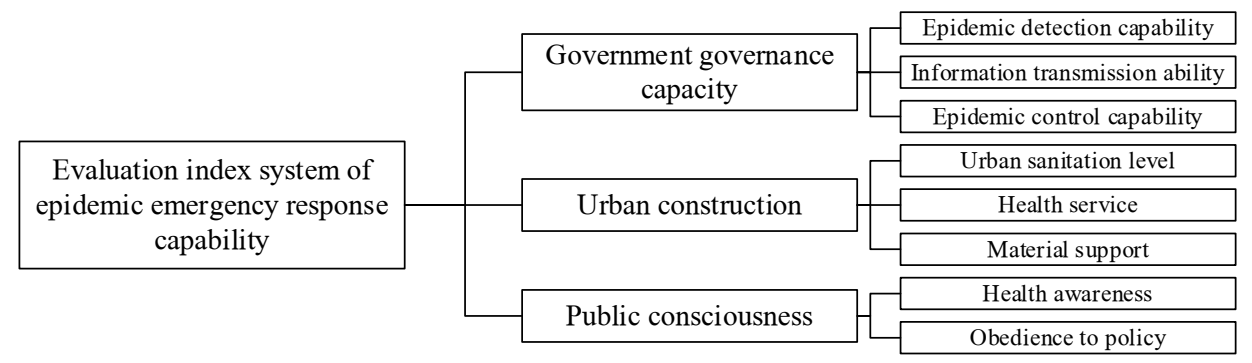

Fig. 1. Index analysis system.

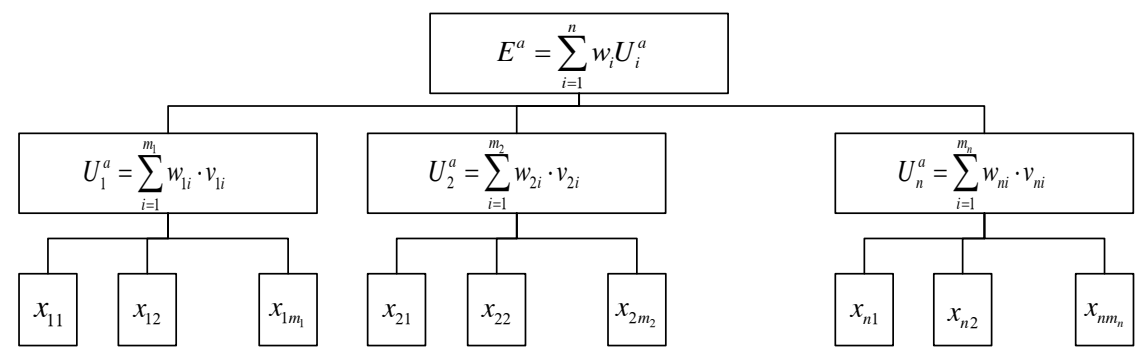

Fig. 2. Mathematical description of index system.

\subsection{Assessment method}

Based on the evaluation model established in section 1.2, this section introduces the specific evaluation methods.

\subsubsection{Establish evaluation matrix}

The above indices are qualitative indices, so we decide to construct a set includes " $\{$ excellent, good, medium, poor $\}$ ". And many experts in this field are invited to evaluate the level of these indices. Then, each evaluation level is quantified, and the rules are shown in Table 1.

Table 1. Quantization table

\begin{tabular}{cllll}
\hline level & excellent & good & medium & poor \\
\hline value & 0.8 & 0.6 & 0.4 & 0.2 \\
\hline
\end{tabular}

The corresponding evaluation matrix will be obtained, as shown below.

$$
V=\left\{v_{1}, v_{2}, \ldots, v_{n}\right\}
$$

\subsubsection{Calculation of the index weight}

Index weighting methods usually includes subjective and objective weighting methods. Based on analytic hierarchy process (AHP), the judgment matrix is calculated. Then, we judge the consistency of judgment matrix by mathematical method. If the consistency degree is lower than a certain threshold, it indicates that there are too many mistakes in the judgment matrix given by the expert. And this expert is required to re-evaluate until the consistency requirements are met. Through this method to adjust the weight value of index, it can increase the credibility of the results.

(1) Calculate the judgment matrix

$C=\left\{c_{1}, c_{2}, \ldots, c_{n}\right\}$ is an index set composed of $\mathrm{n}$ indices, $E=\left\{e_{1}, e_{2}, \ldots, e_{m}\right\}$ is a decision 
group composed of $\mathrm{m}$ experts. The judgement matrix given by the k-th expert is as follows:

$$
A^{k}=\left[\begin{array}{llll}
a_{11}^{k} & a_{12}^{k} & \cdots & a_{1 n}^{k} \\
a_{21}^{k} & \mathrm{a}_{22}^{k} & \cdots & a_{2 n}^{k} \\
\vdots & \vdots & \cdots & \vdots \\
a_{n 1}^{k} & a_{n 2}^{k} & \cdots & a_{n n}^{k}
\end{array}\right]
$$

Among them, $a_{i j}^{k}$ represents the relative weight of index $C_{i}$ and index $C_{j}$. The judgment matrix is defined by the number 1-9 and its reciprocal as a scale.

(2) Calculate the sort vector

In this paper, the arithmetic mean of all column vectors is used as the weight vector. The formula is shown below:

$$
w_{i}^{k}=\frac{1}{n} \sum_{j=1}^{n} \frac{a_{i j}^{k}}{\sum_{i=1}^{n} a_{i j}^{k}} \quad \mathrm{i}=1,2, \ldots, n
$$

Then, the sort vector is $W^{k}=\left(w_{1}^{k}, w_{2}^{k}, \ldots, w_{n}^{k}\right)^{T}$

(3) Check the consistency of matrix

Calculate the completely consistent judgment matrix for $A^{k}$.

$$
\left(A^{*}\right)^{k}=\left[w_{i j}^{k}\right]_{n \times n}, \quad w_{i j}^{k}=\frac{w_{i}^{k}}{w_{j}^{k}}
$$

$A^{*}$ is a completely consistent judgment matrix for $A^{k}$. The relative distance $\xi_{i}^{k}$ is used to measure the difference of each row between matrix $A^{k}$ and matrix $\left(A^{*}\right)^{k}$.

$$
\xi_{i}^{k}=\frac{\sum_{j=1}^{n}\left|a_{i j}^{k}-w_{i j}^{k}\right|}{\sum_{j=1}^{n} w_{i j}^{k}}
$$

The consistency degree of each expert judgment matrix is measured according to the formula (6).

$$
\overline{\xi^{k}}=\frac{\sum_{i=1}^{n} \xi_{i}^{k}}{\sum_{k=1}^{m} \sum_{i=1}^{n} \xi_{i}^{k}}
$$

This paper takes $\overline{\xi^{k}}<0.2$ as the criterion for re-evaluation.

(4) Get the final weight

Through each judgment matrix, the ranking vector is calculated, and the average value is used as the final index weight. 


$$
W=\left(w_{1}, w_{2}, \ldots w_{n}\right), \quad w_{i}=\frac{\sum_{k=1}^{m} w_{i}^{k}}{m}
$$

\subsubsection{Comprehensive evaluation}

After obtaining the capability value of the lower level index, the value of the upper index is obtained through aggregation. In this way, we can get the final evaluation value.

For city $a$, its epidemic response capability is calculated as follows:

$$
E^{a}=\sum_{i=1}^{n} w_{i} U_{i}^{a}
$$

Among them, $U_{i}^{a}$ represents the comprehensive capability value of index $i$ in city $a$, $w_{i}$ is the weight of the composite index.

The comprehensive ability value of index $i$ is:

$$
U_{i}^{a}=\sum_{j=1}^{m} w_{i j} \cdot v_{i j}
$$

Among them, $w_{i j}$ represents the weight of individual indices, $m$ represents the number of indices, and $v_{i j}$ represents the fuzzy value of corresponding indices.

\section{Case analysis}

Four cities participated in the evaluation of advanced cities for epidemic emergency management in region $\mathrm{H}$ of Province $\mathrm{J}$, which mainly included four cities: city A, city B, city $\mathrm{C}$ and city $\mathrm{D}$. Ten experts gave their evaluation values for each index in the indices systems.

In order to evaluate which the best proactive city, we take the combined index includes urban sanitation level, health service and material support and so on. We obtained the evaluation ratings of the indices from these experts. Then, the evaluation values are quantified according to the corresponding rules. The corresponding evaluation matrix is obtained as follows:

$$
V_{2}=\left[\begin{array}{llll}
0.8 & 0.4 & 0.8 & 0.2 \\
0.6 & 0.6 & 0.8 & 0.4 \\
0.8 & 0.4 & 0.6 & 0.4
\end{array}\right]
$$

In addition, these experts give the corresponding judgment matrix for the weight of these three indices, as shown below.

$$
\begin{aligned}
& A_{1}=\left[\begin{array}{lll}
1 & 1 / 6 & 1 / 4 \\
6 & 1 & 2 \\
4 & 1 / 2 & 1
\end{array}\right], A_{2}=\left[\begin{array}{lll}
1 & 1 / 4 & 1 / 2 \\
4 & 1 & 2 \\
2 & 1 / 2 & 1
\end{array}\right], A_{3}=\left[\begin{array}{lll}
1 & 1 / 6 & 1 / 4 \\
6 & 1 & 3 \\
4 & 1 / 3 & 1
\end{array}\right], A_{4}=\left[\begin{array}{lll}
1 & 1 / 5 & 1 / 3 \\
5 & 1 & 2 \\
3 & 1 / 2 & 1
\end{array}\right], A_{5}=\left[\begin{array}{lll}
1 & 1 / 6 & 1 / 2 \\
6 & 1 & 3 \\
2 & 1 / 3 & 1
\end{array}\right] \\
& A_{6}=\left[\begin{array}{lll}
1 & 1 / 5 & 1 / 4 \\
5 & 1 & 2 \\
4 & 1 / 2 & 1
\end{array}\right], A_{7}=\left[\begin{array}{lll}
1 & 1 / 3 & 1 / 2 \\
3 & 1 & 2 \\
2 & 1 / 2 & 1
\end{array}\right], A_{8}=\left[\begin{array}{lll}
1 & 1 / 5 & 1 / 2 \\
5 & 1 & 3 \\
2 & 1 / 3 & 1
\end{array}\right], A_{9}=\left[\begin{array}{lll}
1 & 1 / 7 & 1 / 2 \\
7 & 1 & 2 \\
2 & 1 / 2 & 1
\end{array}\right], A_{10}=\left[\begin{array}{lll}
1 & 1 / 7 & 1 / 4 \\
7 & 1 & 3 \\
4 & 1 / 3 & 1
\end{array}\right]
\end{aligned}
$$

Firstly, formula (5) and formula (6) are used to judge the consistency degree of each judgment matrix, which all meet the consistency requirement.

Then, formula (3) is used to calculate the ranking vectors of the experts. The standard 
matrix formed by the ranking vectors is shown below.

$\left[\begin{array}{llllllllll}0.0893 & 0.1429 & 0.0869 & 0.1096 & 0.1111 & 0.0982 & 0.1638 & 0.1222 & 0.1099 & 0.0796 \\ 0.5869 & 0.5714 & 0.6393 & 0.5813 & 0.6667 & 0.5679 & 0.5390 & 0.6479 & 0.6267 & 0.6555 \\ 0.3238 & 0.2857 & 0.2737 & 0.3092 & 0.2222 & 0.3339 & 0.2972 & 0.2299 & 0.2634 & 0.2648\end{array}\right]$

The weight of each single index under the urban construction is obtained by formula (7).

$$
W_{2}=\left[\begin{array}{lll}
0.1114 & 0.6083 & 0.2804
\end{array}\right]
$$

According to formula (9), the evaluation values of various composite indices in urban construction are obtained as follows:

$$
\begin{aligned}
U_{2} & =\left\{U_{2}^{a}, U_{2}^{b}, U_{2}^{c}, U_{2}^{d}\right\} \\
& =W_{2} \cdot V_{2} \\
& =\{0.6783,0.5217,0.8001,0.3778\}
\end{aligned}
$$

Similarly, the government governance capability and public consciousness of these four cities are calculated by the steps above, and the results are shown below:

$$
\begin{aligned}
& U_{1}=\{0.7430,0.6859,0.6513,0.4795\} \\
& U_{3}=\{0.5749,0.4351,0.6320,0.4751\}
\end{aligned}
$$

In the same way, the weights of the three composite indices of government governance capability, urban construction and public consciousness can be obtained.

$$
W=\left[\begin{array}{lll}
0.3042 & 0.4873 & 0.2085
\end{array}\right]
$$

Finally, formula (8) is used to obtain the composite index value of the emergency capability. The results are shown below:

$$
E=\{0.6764,0.5536,0.7198,0.4290\}
$$

According to the assessment results, city $\mathrm{C}$ has the strongest epidemic emergency response capability and ranks top in this evaluation, while city D lags behind other cities in this evaluation. In order to improve the epidemic emergency response capability of city D, the region $\mathrm{H}$ needs to consider adjusting the allocation of relevant resources.

\section{Conclusion}

In this paper, an evaluation method based on fuzzy comprehensive evaluation was proposed regarding the evaluation of advanced cities for epidemic emergency management in region $\mathrm{H}$, province $\mathrm{J}$. The assessment method was applied to evaluate the emergency response capability of four cities with similar background. After this evaluation, we can find out which city has better emergency response capability easily. Also, policies can be made to improve cities with poor performance. Therefore, this method is meaningful to improve the urban capability.

\section{References}

1. Ya-yun Wei,Jing-yan Zhang,Jia Wang. Research on Building Fire Risk Fast Assessment Method Based on Fuzzy comprehensive evaluation and SVM[J]. Procedia Engineering,2018,211.

2. He Huang,Fu-zhou Luo. Fuzzy comprehensive evaluation for risk analysis of regional machine tool industrial cluster[J]. Systems Engineering Procedia,2011,2.

3. Xu Yihong,Shi Mengju. Fuzzy Comprehensive Evaluation of Beverage Enterprise Risks from System Engineering Perspective[J]. Systems Engineering Procedia,2012,3. 
4. Calistri Paolo,Conte Annamaria,Natale Fabrizio,Possenti Luigi,Savini Lara,Danzetta Maria Luisa,Iannetti Simona, Giovannini Armando. Systems for prevention and control of epidemic emergencies.[J]. Veterinaria italiana,2013,49(3).

5. John-Arne Røttingen,Tore Godal. Speeding up epidemic emergency response[J]. Science,2015,350(6257). 